\title{
Local Voronoi Diagram based Heuristic Anti-Monitoring Method for Moving Objects Traversing through Sensory Fields
}

\author{
Hongbo Huang \\ Information Engineering Institute, Yueyang Vocational Technical College, Yueyang, Hunan, 414006, \\ China
}

Keywords: Sensor network, Anti-monitoring, sensory field, Voronoi diagram.

\begin{abstract}
As for the safety of mobile objects when traversing through a sensory field, a new exposure model based on voronoi diagram is proposed, and furthermore, a new heuristic antimonitoring method for moving objects is designed to find a safe path with the minimum risk when the mobile objects are traversing through the sensory field. Theoretical analysis and simulation results show that, the newly proposed method can achieve satisfying anti-monitoring performance and can select a path with minimum risk for mobile objects to traverse through the sensory field.
\end{abstract}

\section{Introduction}

Sensor networks are proved to be of great potential in a wide variety of commercial and military applications [1-4]. And in some applications, the destination of the mobile objects is to find a safety path with highest possibility to traverse the sensory fields. Therefore, the relationship between the destinations of sensor networks and mobile objects can be described as Monitoring and AntiMonitoring [5].

As for the anti-monitoring problem of mobile objects, many researchers have proposed different methods to help the mobile objects find a path with minimal exposure degree to travel across the sensory field. For example, in reference [6], the authors proposed a path searching algorithm based on grids for the mobile objects, and the exposure degree of a path is defined as the total energy of sensor nodes detected by the mobile object while it moves along the path. In reference [7], the path's exposure degree is defined as the euclidean distance between the sensors and the path. In reference [8], the path's exposure degree is defined as the possibility that the mobile object will be detected by the sensor network when it moves along the path to its destination location. In reference [9], the exposure degree is defined as the signal strength that the sensor network received from the mobile object, and the candidate points set of next hop location is obtained by the voronoi tessellation partition. In reference [10], a new concept of local voronoi tessellation is proposed to confirm the candidate points set of next location.

In this paper, a novel dynamic heuristic anti-monitoring algorithm is proposed based on local voronoi tessellation, in which, the path's exposure degree is defined as the integral signal strength that the mobile object received from all detected sensors when it moves along the path, the candidate points set of next hop location is determined by the local voronoi tessellation partition. Theoretical and simulation results show that, our new algorithm can ensure that the mobile object can move along a path with approximately minimum exposure degree to traverse across the sensory field.

\section{Related Models}

\section{Voronoi Tessellation Model.}

For any two points $P_{1}\left(w_{1}, \xi_{1}\right)$ and $P_{2}\left(w_{2}, \xi_{2}\right)$ on the Earth's surface, let $w_{i}$ and $\xi_{i}$ represent the latitude and longitude of $P_{i}$ respectively, $R$ represent the Earth's radius, then the distance between these two points $P_{1}$ and $P_{2}$ can be represented as

$$
D\left(P_{1}, P_{2}\right)=\frac{\arccos \left(\cos w_{1} \cos w_{2} \cos \left(\xi_{1}-\xi_{2}\right)+\sin w_{1} \sin w_{2}\right)}{360^{\circ}} 2 \pi R
$$


Based on the above formula (1), we can give the definition of VT (Voronoi Tessellation) of a sensor as follows:

Definition 1: Suppose there are a total of $N$ sensors $\left\{S_{1}, S_{2} \ldots S_{N}\right\}$ deployed in a sensory field $F_{S}$, then, the Voronoi Tessellation (VT) of the $i$-th sensor $S_{i}$ can be defined as

\section{Sensing Model.}

$$
V T\left(P_{i}\right)=\left\{x \in F_{S} \mid d\left(x, S_{i}\right)<d\left(x, S_{j}\right), \forall S_{j} \in\left\{S_{1}, S_{2}, \ldots, S_{N}\right\} \text { for } j \neq i\right\}
$$

When a mobile object $O$ is traversing through the sensory field $F_{S}$, for each sensor $S_{i}$ deployed in $F_{S}$, let its sensing radius be $r_{S}$, which means that each sensor can only discover the mobile object within the coverage of its sensing radius $r_{S}$. Since it is obvious that the sensor's sensing ability will diminish with the increasing of the distance from itself to the mobile object, then we can define a general sensing model $\varphi$ for $S_{i}$ as follows:

$$
\varphi\left(O, S_{i}\right)=\frac{\lambda}{\left[d\left(O, S_{i}\right) / r_{S}\right]^{\sigma}}
$$

Where $\lambda$ and $\sigma$ are technology and environment-dependent parameters respectively.

\section{Exposure Model.}

Let $r_{S}=\delta^{*} r_{D}$, where $0<\delta \leq 1$, for a given sensor $S_{i}$, since it is obvious that the exposure probability of the mobile object $O$ will decrease with the increasing of the distance from itself to $S_{i}$, and especially, when the distance is bigger than the sensing radius of the sensors, the detection ability of the mobile object will tend to be 0 , then the exposure probability that the mobile object $O$ will be detected by $S_{i}$ can be defined as

$$
P_{E}\left(O, S_{i}\right)=\left\{\begin{array}{l}
0: \text { if } d\left(O, S_{i}\right)>\delta^{*} r_{D} \\
\varphi\left(O, S_{i}\right): \text { else }
\end{array}\right.
$$

At the time point $t$, suppose that there are a total of $N(t)$ sensors $\left\{S_{1}, S_{2}, \ldots, S_{N(t)}\right\}$ being detected by the mobile object $O$, then, based on the above formula (4), we define the exposure degree of the mobile object $O$ at the time point $t$ as follows:

$$
D_{E}(t)=\sum_{i=1}^{N(t)} P_{E}\left(O, S_{i}\right)
$$

Suppose that the mobile object $O$ is moving in the sensory field $F_{S}$ from the point $P\left(t_{1}\right)$ to $P\left(t_{2}\right)$ along the path $P(t)$, then, according to the above description, we define the general exposure model $\Psi_{E}$ for the mobile object $O$ when traveling along the path $P(t)$ from the point $P\left(t_{1}\right)$ to $P\left(t_{2}\right)$ as follows:

$$
\Psi_{E}\left(P\left(t_{1}\right), P\left(t_{2}\right)\right)=\int_{t_{1}}^{t_{2}} D_{E}(t)\left|\frac{d P(t)}{d t}\right| d t
$$

Where $\left|\frac{d P(t)}{d t}\right|$ represents the element of arc length, and let $L\left(P\left(t_{1}\right), P\left(t_{2}\right)\right)$ represent the length of the path $P(t)$ along which the mobile object $O$ moves from the point $P\left(t_{1}\right)$ to $P\left(t_{2}\right)$, then we have

$$
L\left(P\left(t_{1}\right), P\left(t_{2}\right)\right)=\int_{t_{1}}^{t_{2}}\left|\frac{d P(t)}{d t}\right| d t
$$

\section{Voronoi Tessellation based Algorithm}

\section{Cost Function Design for the Next Hop Selection.}

For the mobile object $O$, suppose that its starting position is $S$, its destination position is $D$, its current position is $C$, and its next hop position is $x$, then our newly proposed scheme shall be able to guarantee that both the values of $\Psi_{E}(S, D)=\Psi_{E}(S, C)+\Psi_{E}(C, x)+\Psi_{E}(x, D)$ and $L(S, D)$ $=L(S, C)+L(C, x)+L(x, D)$ may be as small as possible.

Considering $\Psi_{E}(S, D)=\Psi_{E}(S, C)+\Psi_{E}(C, x)+\Psi_{E}(x, D)$ and $L(S, D)=L(S, C)+L(C, x)+L(x, D)$, it is obvious that both $\Psi_{E}(S, C)$ and $L(S, C)$ are known values for the mobile object $O$, but the values of 
$\Psi_{E}(C, x), \Psi_{E}(x, D), L(C, x)$ and $L(x, D)$ need to be estimated by the mobile object $O$ according to the environment of its detected sensory area.

Let $L_{0}(t)$ represent the sensory area in which the deployed sensors can be detected by the mobile object $O$ at the time point $t, L_{1}(t)$ represent the sensory area in which the deployed sensors cannot be detected by the mobile object $O$ at the time point $t$ but have distance to $P$ less than or equaling to $r_{S}, S\left(L_{0}, t\right)$ represent the size of $L_{0}(t), S\left(L_{1}, t\right)$ represent the size of $L_{1}(t)$, then we have

$$
\begin{aligned}
& S\left(L_{0}, t\right)=\left\{\begin{array}{l}
0: \text { if } \quad d(O, P)>r_{D} \\
\pi * \delta^{2 *} r_{D}^{2}: \text { elseif } \quad 0 \leq d(O, P) \leq r_{D}-\delta * r_{D} \\
\alpha_{1}^{*} r_{D}^{2}+\alpha_{2}{ }^{*} \delta^{2}{ }^{*} r_{D}^{2}-r_{D} * d(O, P) * \sin \alpha_{1}: \text { otherwise }
\end{array}\right. \\
& S\left(L_{1}, t\right)=\left\{\begin{array}{l}
\pi * \delta^{2 *} r_{D}^{2}: \text { if } \quad d(O, P)>r_{D} \\
0: \text { elseif } \quad 0 \leq d(O, P) \leq r_{D}-\delta^{*} r_{D} \\
\pi^{*} \delta^{2 * r_{D}}{ }^{2}-\alpha_{1}^{*}{ }_{D}^{2}{ }_{D}^{2}-\alpha{ }^{*} \delta^{2 *} r_{D}^{2}+r_{D} * d(O, P) * \sin \alpha_{1}: \text { otherwise }
\end{array}\right.
\end{aligned}
$$

Where

$$
\begin{aligned}
& \alpha_{1}=\arccos \frac{r_{D}^{2}+d^{2}(O, P)-\delta^{2 *} r_{D}^{2}}{2 * r_{D} * d(O, P)} \\
& \alpha_{2}=\arccos \frac{\delta^{2 *} r_{D}^{2}+d^{2}(O, P)-r_{D}^{2}}{2 * \delta^{*} r_{D} * d(O, P)}
\end{aligned}
$$

At the time point $t$, suppose that the mobile object $O$ has detected $N_{0}(t)$ sensors $\left\{S_{1}, S_{2} \ldots S_{N_{0}(t)}\right\}$ deployed in the sensory area $L_{0}(t)$, then the deployment density of sensors in the sensory area $L_{0}(t)$ can be estimated as

$$
D\left(L_{0}, t\right)=N_{0}(t) / S\left(L_{0}, t\right)
$$

Since the area $L_{1}(t)$ is adjacent to the area $L_{0}(t)$, let $N_{1}(t)$ represent the number of sensors deployed in $L_{1}(t)$, then $N_{1}(t)$ can be estimated as

$$
N_{1}(t)=D\left(L_{0}, t\right)^{*} S\left(L_{1}, t\right)
$$

Hence, the exposure degree of the mobile object $O$ at the location point $P$ and the time point $t$ can be estimated as:

$$
D_{E}(P, t)=\sum_{i=1}^{N_{0}(t)} P_{E}\left(P, S_{i}\right)+\frac{N_{1}(t)+1}{N_{0}(t)+1} * \sum_{i=1}^{N_{0}(t)} P_{E}\left(P, S_{i}\right)
$$

Therefore, when the mobile object $O$ is moving from its current location point $C$ (let $C=P\left(t_{1}\right)$ ) to its next location point $x$ (let $x=P\left(t_{2}\right)$ ) along the straight line path $P(t)$ between $C$ and $x$, the exposure $\Psi_{E}(C, x)$ of the mobile object $O$ can be estimated as follows:

$$
\Psi_{E}(C, x)=\int_{t_{1}}^{t_{2}} D_{E}(P(t), t)\left|\frac{d P(t)}{d t}\right| d t
$$

Next, according to the formula (7), we have

$$
L(C, x)=\int_{t_{1}}^{t_{2}}\left|\frac{d P(t)}{d t}\right| d t
$$

And then, for convenience, since the sensory field between the next location points $x$ of the mobile object $O$ and its destination point $D$ is unknown to $O$, then $\Psi_{E}(x, D)$ and $L(x, D)$ can be simply estimated as follows:

$$
\begin{gathered}
L(x, D)=d(x, D) \\
\Psi_{E}(x, D)=\frac{L(x, D) *\left(\Psi_{E}(S, C)+\Psi_{E}(C, x)\right)}{L(S, C)+L(C, x)+L(x, D)}
\end{gathered}
$$

Finally, in order to guarantee that our dynamic heuristic path searching scheme can select a suitable next hop position $x$ dynamically for the mobile object $O$ at the current time point $t$, we define a cost function $f(x)$ as follows:

$$
f(x)=g(x)+\omega^{*} h(x)
$$

Here, $0 \leq \omega \leq 1$ and for $0 \leq \lambda_{1} \leq 1$ and $0 \leq \lambda_{2} \leq 1$, there are 


$$
\begin{aligned}
& g(x)=\lambda_{1} * \frac{\Psi_{E}(C, x)}{\Psi_{E}(S, C)+\Psi_{E}(C, x)+\Psi_{E}(x, D)}+\left(1-\lambda_{1}\right) * \frac{L(C, x)}{L(S, C)+L(C, x)+L(x, D)} \\
& h(x)=\lambda_{2} * \frac{\Psi_{E}(x, D)}{\Psi_{E}(S, C)+\Psi_{E}(C, x)+\Psi_{E}(x, D)}+\left(1-\lambda_{2}\right) * \frac{L(x, D)}{L(S, C)+L(C, x)+L(x, D)}
\end{aligned}
$$

\section{Selection Method for the Set of Candidate Next Hop Positions.}

According to the above analysis, it is obvious that if we want to make our scheme work, we shall provide a suitable set of candidate next hop positions for the mobile object $O$ at its current location. Supposing that the mobile object $O$ is moving across the sensory field from the source location $S$ to its destination location $D$, then, it may meet the following three kinds of different conditions at its current location $C$ :

1. No sensors are detected by $O$ : it is easy to know that the mobile object $O$ can move from $C$ to $D$ along the straight line segment between $C$ and $D$. Thus, in this condition, we can simply select the set $\{P\}$ as $O$ `s current candidate set of the next hop positions.

2. Only one sensor $S_{1}$ is detected by $O$ : it is obvious that the mobile object $O$ can move along the circumference's periphery of $c\left(S_{1}, r_{D}\right)$ to the nearest tangency point $P_{1}$ between $D$ and the circumference of $c\left(S_{1}, r_{D}\right)$ first, and then move from the tangency point $P_{1}$ to $D$ along the straight line between $P_{1}$ and $D$. Since there exist two tangency points $P_{1}$ and $P_{2}$ between $D$ and the circumference of $c\left(S_{1}, r_{D}\right)$, therefore, we can simply select the set $\left\{P_{1}, P_{2}\right\}$ as $O$ 's current candidate set of the next hop positions.

3. Two or more sensors are detected by $O$ : supposing that there are $n$ active sensors $\left\{S_{1}, S_{2}, \ldots, S_{n}\right\}$ detected by the mobile object $O$ at its current location $C$, it is obvious that $O$ can compute out the voronoi tessellation of $c\left(C, r_{D}\right)$ according to the formula (2) first, and then, we can simply select the set of intersection points $\left\{P_{1}, P_{2}, \ldots, P_{n}\right\}$ between the voronoi tessellation and the borderline of the mobile object's detection area $c\left(C, r_{D}\right)$ as $O$ 's current candidate set of the next hop positions. Since from the formula (2) to the formula (6), it is easy to know that the boundary lines of the voronoi tessellation are the safest paths, therefore, the mobile object $O$ can select a suitable point $P_{i}$, which satisfies $f\left(P_{i}\right)=$ minimum $\left\{f\left(P_{1}\right), f\left(P_{2}\right), \ldots, f\left(P_{n}\right)\right\}$, from $\left\{P_{1}, P_{2}, \ldots, P_{n}\right\}$ based on the cost function given in the formula (19) first, and then move along the boundary lines of the voronoi tessellation to $P_{i}$ next, and thereafter, move from the point $P_{i}$ to $D$ along the straight lin $\mathrm{x}$ between $P_{i}$ and $D$ finally.

\section{New Algorithm based on Voronoi Tessellation.}

According to above descriptions, we can present our new Heuristic Anti-Monitoring Algorithm based on Voronoi Tessellation (HAMA-VT for abbreviation) as follows:

Step 1: According to the formula (2), the mobile object $O$ computes out its voronoi tessellation.

Step 2: According to the method given in the section 3.2, the mobile object $O$ computes out its candidate set of the next hop positions.

Step 3: According to the formula (19), the mobile object $O$ selects out a suitable point $P$, which satisfies $f\left(P_{i}\right)=$ minimum $\left\{f\left(P_{1}\right), f\left(P_{2}\right), \ldots, f\left(P_{n}\right)\right\}$, from its candidate set of the next hop positions.

Step 4: First, the mobile object $O$ moves along the boundary lines of the voronoi tessellation to the point $P$, and then moves from the point $P$ to $D$ along the straight line between $P_{i}$ and $D$.

Let the time interval of each decision round be $\mathrm{T}$, then, at the start time of each decision round, the mobile object $O$ will turn back to the step 1 and execute the whole algorithm HAMA-VT again.

\section{Experimental Result and Theoretical Analysis}

In order to evaluate the performance of the newly proposed algorithm HAMA-VT, we have done a wide range of simulations. The simulation program is coded by Java and implemented in system environment with Pentium 4 3.06G, 1GB and WINDOWS XP. For simplicity, the sensory field in our simulation is defined as a 500*500 square, and sensors are randomly deployed in the region. The mobile object $O$ needs to move from the source location $S(20,20)$ to the target location $D$ $(480,480)$. 
Our first experimental item is to explore the effects of the detection radius $r_{D}$ of the mobile object to the path searching performance of the algorithm HAMA-VT. For simplicity, the parameters $\lambda, \sigma$, $\omega, \lambda_{1}$ and $\lambda_{2}$ are set to be $0.05,1,0.5,0.5$ and 0.5 respectively. The simulation results are illustrated in the following Fig.1 to Fig.2.

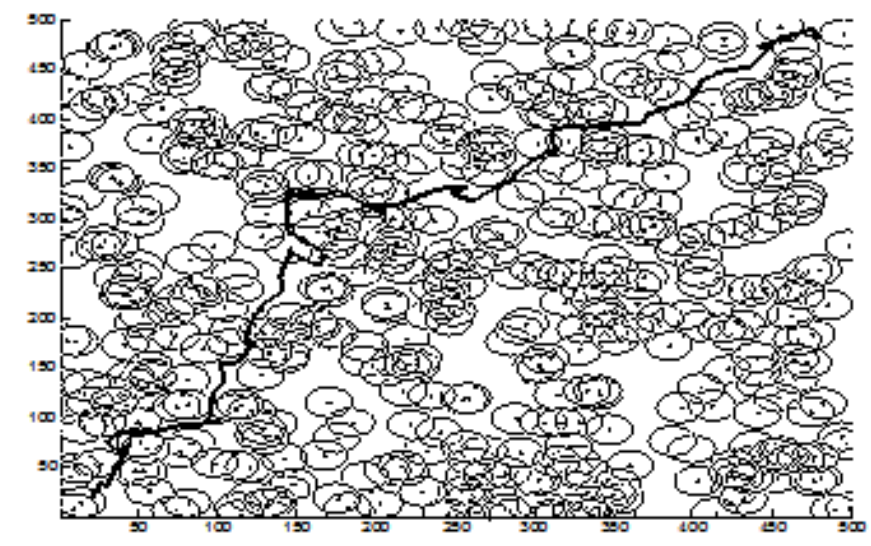

Fig. $1 r_{D}=80$

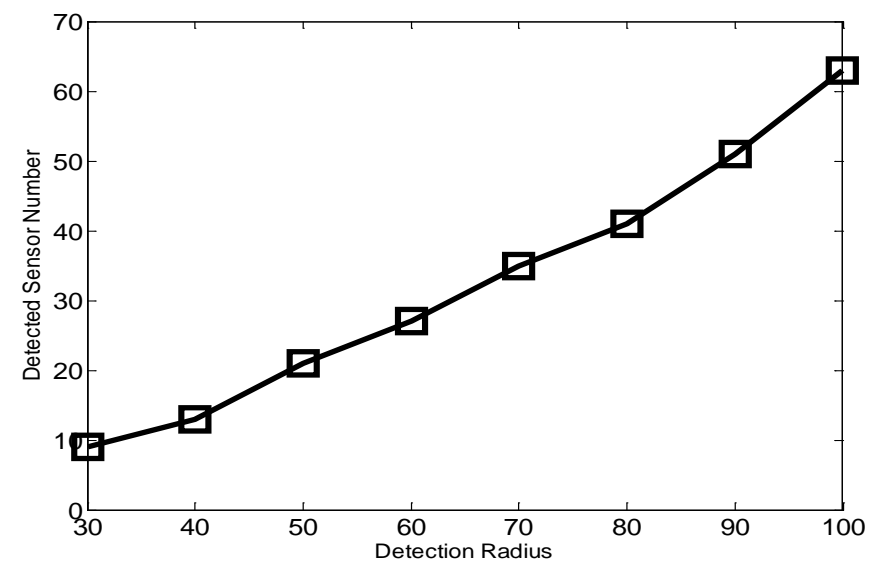

Fig.2 relationship between $\mathrm{rD}$ and the number of detected sensors

\section{Conclusions}

Considering the anti-monitoring problem of the mobile object in the sensory field, in this paper, we have designed a new exposure model, a new cost function, and a new dynamic heuristic antimonitoring path searching algorithm for the mobile object to traverse across the sensory field safely. Experimental results show that, the new distributed algorithm has good anti-monitoring performance, and can ensure the mobile object to find an approximately most safety path to traverse across the sensory field. For future works, we aim to do more research on three dimensional anti monitoring problem to meet the applications in three dimensional space.

\section{References}

[1] I. F. Akyildiz, Weilian Su, Y. Sankarasubramaniam and E. Cayirci, A Survey on Sensor Networks, IEEE Communications Magazine, 40(8), pp.102-114, 2002.

[2] Lei Wang, Ruizhong Wei, Yaping Lin, Bo Wang. A Clique based Node Scheduling Method for Wireless Sensor Networks. Journal of Network and Computer Applications. 33(4), pp.383-396, 2010.

[3] Lei Wang, Ruizhong Wei. Reputation Model based Pair-wise Key Establishment Scheme for Sensor Networks. Ad Hoc \& Sensor Wireless Networks. 9(3-4), pp.163-177, 2010. 
[4] Chen Tao, Yang Zheng, Liu Yunhao, Guo Deke. Localization-Oriented Network Adjustment in Wireless Ad Hoc and Sensor Networks. IEEE Transactions on Parallel and Distributed Systems, 25(1), pp.146-155, 2014.

[5] Wenzhe Zhang, Minglu Li, Wei Shu, Min-You Wu, Smart Path-Finding with Local Information in a Sensory Field, In Proc. of the Second International Conference on Mobile Ad-hoc and Sensor Networks, Hong Kong, China, pp.119-130, December, 2006.

[6] S. Meguerdichian, F. Koushanfar, G. Qu, and M. Potkonjak, Exposure in wireless ad-hoc sensor networks, In Proc. of the ACM MobiCom, pp.139-150, 2001.

[7] S. Meguerdichian, F. Koushanfar, M.Potkonjak, et al. Coverage problems in wireless ad-hoc sensor networks, In Proc. of the IEEE INFOCOM, 3, pp.1380-1387, 2001.

[8] V. Phipatanasuphorn and Parameswaran Ramanathan, Vulnerability of Sensor Networks to unauthorized Traversal and Monitoring, IEEE Transactions on Computers, 53(3), pp.364-369, 2004.

[9] Veltri G, Huang Q, Qu G, et al. Minimal and maximal exposure path algorithms for wireless embedded sensor networks. In Proc. of the ACM Int'l Conf. on Embedded Networked Sensor Systems (SenSys). New York: ACM Press, pp.40-50, 2003.

[10] W. Z. Zhang, M. L. Li and M. Y. Wu, An Algorithm for Target Traversing Based on Local Voronoi Diagram, Journal of Software, 18(5), pp.1246-1253, 2007. 